\title{
Public key cryptography for quality assurance in randomization for clinical trials
}

Travis C. Porco ${ }^{1,2,3^{*}}$

Nicole E. Stoller ${ }^{1}$

Jeremy D. Keenan ${ }^{1,2,3}$

Robin L. Bailey ${ }^{4}$

Thomas M. Lietman ${ }^{1,2,3}$

1 Francis I. Proctor Foundation for Research in Ophthalmology, Box 0412, University of California, San Francisco, California 94143-0412 USA

2 Department of Epidemiology and Biostatistics, University of California, San Francisco, San Francisco, California, USA

3 Department of Ophthalmology, University of California, San Francisco, San Francisco, California, USA

4 London School of Hygiene and Tropical Medicine, London, England, UK

* To whom correspondence should be directed: Travis C. Porco, Address, email

travis . porco@ucsf . edu; phone (415) 476-4101, FAX (415) 476-0527.

Correspondence; 500 words, 5 references

Key words: clinical trial, randomization, public key cryptography, quality assurance 


\section{Public key cryptography for quality assurance in randomization for clinical trials}

Trusted parties are needed to conduct principled randomizations for individual and community randomized trials. Such randomizations yield assignments statistically independent of all covariates, but should not be influenced by anyone for any reason. The community randomized COMMIT trial allocated treatment assignments using a roulette wheel in a large public ceremony witnessed by all[1], which is unsuitable when masking must be maintained. Commonly, a trusted person simply chooses a randomization list by a method of her or his own choosing. Yet why rely entirely on trust, when verification is possible?

One approach is to initialize a pseudorandom number generator by a starting value, or seed, using a method entirely out of the control of the person conducting the randomization. Once the starting value is chosen, any randomization procedure can be derived from it. Different seeds provide different randomizations, and investigators must thus be masked to this seed.

We propose the use of public key cryptography[2,3] as a simple and inexpensive way to maintain the masking. Public key cryptography is based on creation and use of both public and private keys, so that information encrypted with one key can only be decrypted with the other. Such methods are widely used for secure electronic communication and digital signatures. First, the person responsible for randomization generates a public-private key pair, distributing the public key to all interested parties. The private key is kept secret. Such key generation can be done quickly using OpenSSL[4], freely available on all major platforms. In addition, where applicable, distribution of a timestamped digital hash[3] of the randomization script or program prevents the script itself from being further edited, since any changes would alter this hash.

Next, the responsible person will obtain digital information in a way that prevents influence or cherry-picking. A simple solution which avoids trusted third parties is to record a timestamped agreement to use unpredictable data which do not exist yet. Examples include future reported high temperatures in specific cities, or the top left article in tomorrow's edition of a specified newspaper.

When this information becomes available, the responsible person encrypts it (or a digital hash of it) with the private key. This encrypted information becomes the random number seed, and so must be kept secret. Upon unmasking after study completion, the authenticity of the random number seed can be checked by applying the public key already in possession. Neither the private key nor the seed can have been altered. Yet during the trial, possession of the public key and the unencrypted message do not reveal the seed, produced by encryption with the private key.

We conducted a pilot of this method for the Malawi portion of the Mortality Reduction after Oral Azithromycin Trial (clinicaltrials.gov identifiers NCT02047981 and NCT02048007), a new double masked, placebo controlled, cluster randomized clinical trial testing whether mass administration of azithromycin reduces mortality in young children (as suggested in an earlier trial[5]). Communities including approximately 85,000 children are being enrolled at the Malawi site, and the intervention is planned for two years. 


\section{References}

[1] Gail MH, Byar DP, Pechacek TF, Corle DK. Aspects of statistical design for the community intervention trial for smoking cessation (COMMIT). Control Clin Trials 1990; 13:6-21.

[2] Batten LM, Public Key Cryptography: applications and attacks. Piscataway, NJ: Wiley/IEEE Press; 2013.

[3] Katz J, Lindell Y. Introduction to Modern Cryptography. 2d ed. Boca Raton, FL: Taylor and Francis/CRC Press; 2014.

[4] OpenSSL. https : / /www . openss l . org. Accessed: 27 Feb 2014.

[5] Porco TC, Gebre T, Ayele B, House J, Keenan J, Zhou Z, et al. Effect of mass distribution of azithromycin for trachoma control on overall mortality in Ethiopian children: a randomized trial. JAMA 2009; 302(9):962-8. 


\section{Acknowledgments}

T. Porco, J. Keenan, N. Stoller, R. Bailey, and T. Lietman acknowledge funding from the Bill and Melinda Gates Foundation trial for Mortality Reduction after Oral Azithromycin (T. Lietman, PI), a suite of cluster randomized trials (clinicaltrials.gov identifiers NCT02047981 and NCT02048007). The funding agency played no role in the design, analysis, or decision to publish. 